Research Article

\title{
Investigation of the Mechanism of Zishen Yutai Pills on Polycystic Ovary Syndrome: A Network Pharmacology and Molecular Docking Approach
}

\author{
Yingyin Chen $\mathbb{D}^{1,2}$ Xinyi Chai $\mathbb{D}^{1,}{ }^{1}$ Ying Zhao $\mathbb{D}^{1,3,4}{ }^{1,3 i n q i a n}$ Yang, ${ }^{4}$ Caiting Zhong, \\ and Yihui Feng 1 1,3,4 \\ ${ }^{1}$ Guangzhou University of Chinese Medicine, Guangzhou 510405, China \\ ${ }^{2}$ Lingnan Medical Research Center, Guangzhou University of Chinese Medicine, Guangzhou 510405, China \\ ${ }^{3}$ Department of Gynecology, First Affiliated Hospital of Guangzhou University of Chinese Medicine, Guangzhou 510405, China \\ ${ }^{4}$ First Clinical Medical College of Guangzhou University of Chinese Medicine, Guangzhou 510405, China
}

Correspondence should be addressed to Yihui Feng; gynyhvon@hotmail.com

Received 2 July 2021; Revised 30 October 2021; Accepted 26 November 2021; Published 16 December 2021

Academic Editor: Raffaele Capasso

Copyright $\odot 2021$ Yingyin Chen et al. This is an open access article distributed under the Creative Commons Attribution License, which permits unrestricted use, distribution, and reproduction in any medium, provided the original work is properly cited.

Background. Zishen Yutai Pills (ZSYTP) is a prescription based on traditional Chinese medicine used to treat kidney-deficient pattern in traditional Chinese medicine. It is also widely used clinically for the treatment of polycystic ovary syndrome (PCOS) with positive results. This study aims to explore the potential pharmacological mechanism of ZSYTP for the treatment of PCOS by a network pharmacology approach. Methods. Compounds were collected from the Traditional Chinese Medicine Systems Pharmacology Database and Analysis Platform and Bioinformatics Analysis Tool for Molecular mechanism of Traditional Chinese Medicine and TCM Database@ Taiwan, and the corresponding targets were retrieved from PubChem, Swiss Target Prediction, STITCH, and DrugBank. Meanwhile, PCOS targets were retrieved from the GeneCards database, the Online Mendelian Inheritance in Man database, National Center for Biotechnology Information Database, and DrugBank. Subsequently, multiple network construction and gene enrichment analyses were conducted with Cytoscape 3.8.2 software. Based on the previous results in the study, molecular docking simulations were done. Results. 205 active compounds and 478 ZSYTP target genes were obtained after screening by ADME consideration. 1881 disease-related targets were obtained after removing duplicates. 148 intersection target genes between drug and disease targets were isolated. Gene ontology enrichment analysis and Kyoto Encyclopedia of Genes and Genomes analysis highlighted multiple gene functions and different signaling pathways to treat PCOS. Further molecular docking demonstrated the practicality of in vivo action of ZSYTP to a certain extent. Conclusions. It is possible that the pharmacological effect of ZSYTP on PCOS is linked to the hypoxia-inducible factor 1 (HIF-1) signaling pathway, improving insulin resistance, the variation on gene expression such as RNA splicing, and regulation of mRNA metabolic process. This study paves the way for further research investigating its mechanisms.

\section{Background}

Polycystic ovary syndrome (PCOS) is a common gynecological disorder affecting $5 \%-15 \%$ of women of reproductive age from all over the world [1]. Multiple aspects of a women's holistic health and its long-term effects are affected by PCOS. As one of the most common endocrine-reproductive-metabolic disorders, it affects reproductivity in females and is linked to endocrine disorders like insulin resistance and type 2 diabetes. PCOS patients are known to have an increased risk of cardiovascular disorders such as atherogenic dyslipidemia syndrome, coronary heart disease, and cerebrovascular morbidity. Beyond that, it also influences PCOS patients' mental health [2].

At present, due to the relatively unknown etiology of PCOS, there is no effective treatment protocol. Symptomatic treatment is mainly used in clinical practice, and long-term health management is needed. Traditional Chinese medicine 
(TCM) has long been utilized to treat infertility for centuries and has been proven effective in managing the symptoms of PCOS patients and promoting ovulation. In accordance with TCM theory, PCOS patients who have obesity or insulin resistance [3] can be widely categorized into several TCM patterns, kidney deficiency and phlegm, kidney deficiency and blood stasis, and kidney deficiency or insufficiency of both the spleen and the kidney [4]. It can be observed that most PCOS patients are of a kidney-deficient pattern. Zishen Yutai Pill (ZSYTP) is a patented formula used clinically for kidney-deficient-pattern patients [5]. Further studies have also shown that ZSYTP improved ovulatory functions in patients of this pattern. While the clinical efficacies of ZSYTP have been proven with multiple studies [6], the pharmacological mechanism of its therapeutic effect remains unclear.

In recent years, increasing research has been done on traditional or patented TCM formulae, and it can be a gift for drug discovery [7]. For instance, the study of the known TCM herb Artemisia carvifolia led to the discovery of artemisinin and subsequently became crucial in the fight against malaria [8].

A network pharmacology approach was chosen to uncover ZSYTP's pharmacological mechanism on PCOS, aiming to provide a protocol for studying patented formulae and a specific disease. This study also would provide the data and direction for further clinical trials to develop, improving the efficacy of future drug development studies.

\section{Methods and Materials}

\subsection{Data Preparation}

2.1.1. Screening for Chemical Compounds of Each Herb in ZSYTP. Chemical compounds from 15 herbs in ZSYTP were obtained from the Traditional Chinese Medicine System Pharmacology Database [9] (TCMSP ${ }^{\mathrm{TM}}$, https://old. tcmsp-e.com/tcmsp.php), a unique systemic pharmacology platform devised for Chinese herbal medicines.

The relationships between drugs, targets, and diseases were obtained from a Bioinformatics Analysis Tool for Molecular mechanism of Traditional Chinese Medicine [10] (BATMAN-TCM, http://bionet.ncpsb.org.cn/batman-tcm/ ), which is a bioinformatics analysis tool specially designed for the research of the molecular mechanism of TCM and TCM Database@ Taiwan [11] (http://tcm.cmu. edu.tw/zh-tw/), the world's largest noncommercial TCM database.

Screening filters used in this study were $\mathrm{OB} \geq 30 \%$ and $\mathrm{DL} \geq 0.18$ to maximize drug discovery. These active compounds were included in accordance with ADME consideration (Adsorption, Distribution, Metabolism, Excretion) which could ensure higher efficiency to the active compounds selected.

2.1.2. Screening Target Genes of Each Herb in ZSYTP. We collated IUPAC International Chemical Identifier (InChi) and Canonical SMILES of each 205 chemical compounds of ZSYTP from PubChem database [12] (http:// pubchem.ncbi.nlm.nih.gov/) to corroborate the uniqueness of these molecules in the database we built.

To predict interactions between chemicals and proteins, we input all molecular information of the 205 active compounds into STITCH [13] (http://stitch.embl.de) and Swiss Target Prediction [14] (http://www.swisstargetprediction. ch/index.php). PubChem database was utilized to provide drug-target identification. Finally, we found information on known drugs and their corresponding target genes in DrugBank [15](https://go.drugbank.com/), which is a pharmaceutical knowledge base.

2.1.3. PCOS Targets Database Building. "Polycystic Ovary Syndrome" is the keyword used to retrieve relevant action targets. To obtain more comprehensive disease-related targets, 3 online databases were utilized, including the GeneCards database [16] (http://www.genecards.org/), the Online Mendelian Inheritance in Man database [17] (OMIM, http://www.omim.org/), National Center for Biotechnology Information database [18] (NCBI, https://www. ncbi.nlm.nih.gov/), and DrugBank database (http://www. drugbank.ca/). GeneCards, OMIM, and NCBI database provide comprehensive data on predicted and annotated human genotypes, while DurgBank offers data on the disease and the drugs available to it. Due to the nonstandard naming of the proteins collated from the 3 databases, we used the UniProt database [19] (http://www.uniprot.org/) to match the standard gene names.

\subsection{Network Construction}

2.2.1. Network Construction Method. The network construction was built as follows: (1) ZSYTP active compoundpotential target network (C-T network), (2) PPI network of PCOS disease targets, (3) PPI network of ZSYTP drug targets, and (4) PPI network of interaction between PCOS disease and drug targets.

Venny 2.1 (https://bioinfogp.cnb.csic.es/tools/venny/), an online program, was utilized to visualize and isolate overlapping targets between the active ZSYTP compounds and those related to PCOS. The data were imported into Cytoscape 3.8.2 software to construct the active compoundpotential target network (C-T network). The nodes represent the target genes and compounds, and the line edges represent the correlations between them. The size and transparency of the nodes were set to reflect the degree value. The active compounds and target with the highest degrees were selected for future molecular docking analysis.

PCOS disease targets and ZSYTP drug targets were imported into Cytoscape 3.8.2 software Biogenet add-in [20] to obtain Protein-Protein Interaction (PPI) networks, respectively. Both PPI networks were intersected for further topological screening.

2.2.2. Gene Ontology and KEGG Enrichment Analysis. Cytoscape 3.8.2 software ClueGO 2.5.7 plug-in [21] was used to perform gene ontology (GO) enrichment analysis and 
Kyoto Encyclopedia of Genes and Genomes (KEGG) pathway enrichment analysis. Species was set as "Homo sapiens," and a $p$-value $<0.05$ was set as statistically significant.

2.3. Confirmation of Molecular Docking. Molecular docking simulations were utilized to verify the binding of the target and the corresponding compound. Data about the construction of the macromolecular protein target receptors, including the first eight high degree values which come from the C-T network, were acquired via the RCSB PDB database [22] (PDB, http://www.rcsb.org/), and the small-molecule compounds which are considered as part of the first five high degree value coming from $\mathrm{C}$-T network were retrieved via the PubChem database and TCMSP. Expulsion of water and ligand from macromolecular protein downloaded from PDB was performed using PyMOL 2.4 software [23], and format conversion was performed using Open Babel software [24]. Molecular docking simulations of the macromolecular protein target and the corresponding compounds were carried out by AutoDockTool 1.5.6 and AutoDock 4.2.6 software [25]. The genetic algorithm of the search parameters was used. Visualizing the results was utilized by PyMOL 2.4 software.

The workflow of our study is shown by flowchart (Figure 1).

\section{Results}

3.1. Screened Chemical Compounds of Each Herb in ZSYTP. 1364 chemical compounds in ZSYTP were found in this process; there are 190 from Panax ginseng (RS), 188 from Lycii Fructus (GQZ), 174 from Morindae officinalis Radix (BJT), 165 from Amomum aurantiacum (SR), 135 from Folium Artemisiae Argyi (AY), 134 from Codonopsis Radix (DS), 119 from Eucommiae Cortex (DZ), 76 from Rehmanniae Radix Praeparata (SDH), 55 from Atractylodes Macrocephala Koidz (BZ), 46 from Herba Taxilli (SIS), 30 from Dipsaci Radix (XD), 29 from Cuscutae Semen (TSZ), 17 from Fallopia multiflora (HSW), 4 from Colla Corii Asini (EJ), and 2 from Cornu Cervi Degelatinum (LJS).

Upon application of ADME screening thresholds of $\mathrm{OB} \geq 30 \%$ and $\mathrm{DL} \geq 0.18 \%, 187$ chemical compounds were obtained: 22 from RS, 45 from GQZ, 20 from BJT, 10 from SR, 9 from AY, 21 from DS, 28 from DZ, 2 from SDH, 9 from BZ, 2 from SJS, 6 from XD, 11 from TSZ, and 2 from HSW. LJS and EJ were not found in any online TCM databases; however, further 18 compounds with lower OB or DL values were consolidated as they hold extensive pharmacological activities, 10 from HSW, 4 from EJ, and 2 from LJS. These 205 compounds were considered as active compounds with good biological activity.

3.2. Active Target Genes Prediction of ZSYTP. 205 active compounds were based on molecular similarity to predict the target genes, which was conducted by importing each unique molecular data into STITCH, Swiss Target
Prediction, PubChem, and DrugBank. 478 target genes of every herb of ZSYTP were acquired after eliminating duplication.

3.3. Intersection Targets of ZSYTP and PCOS. A total of 1881 PCOS disease-related targets were obtained upon removing duplicates. Further comparison was made between the 478 potential target genes of ZSYTP and 1881 PCOS disease targets; 148 intersection potential target genes that act on PCOS (Figure 2) were subsequently isolated. Of note, while there are multiple common targets between each herb to disease, target genes are known to have multipathway effects.

3.4. Constructed ZSYTP Compound-Potential Target Network (C-T Network). C-T network illustrates the relationship among each herb, its corresponding active compounds, and targets. These were mapped out by the Cytoscape 3.8.2 software. The nodes describe herbs, active compounds, and target genes, and the edges indicate the relevance among them. A C-T network can provide further insights into the study of ZSYTP's therapeutic mechanism on PCOS.

The constructed C-T network of 39 active compounds includes 200 nodes and 540 edges depicted in Figure 3.

Compounds and targets with the highest degree values were utilized to do molecular docking verification. The degree values are listed in Table 1.

3.5. Core Network Analysis. PPI network of the intersectional potential target genes between disease and drug targets was constructed with Cytoscape version 3.8.2 BisoGenet plug-in.

8937 nodes and 200243 edges were established. Based on a previous study, topological feature analysis targets were selected with parameters above twice the median value. Upon the first screening process, a network of 2469 nodes and 101184 edges was derived. The second selection criteria were based on "Betweenness," "Closeness," "LAC," and "Neighborhood Connectivity"; 473 nodes and 10130 edges were identified. The topological screening process is presented in Figure 4.

3.6. Gene Ontology and KEGG Analysis. Cytoscape 3.8.2 software ClueGO plug-in was utilized to explore the active process of ZSYTP on PCOS by the 473 relationship genes from the PPI network, and $p$ values less than 0.05 were defined as statistically significant. The visualization in this part is shown in Figure 5.

3.6.1. Gene Ontology Biological Process (GO-BP). The enrichment analysis of BP was completed with the Cytoscape ClueGO plug-in for visualization (Figure 5(a)), with $p$-values less than 0.05 as statistically significant. 540 processes were obtained. The top 20 biological processes with ascending $p$ values were selected for further analysis.

The biological processes selected were mainly involved in RNA splicing, cellular macromolecule metabolic process, 


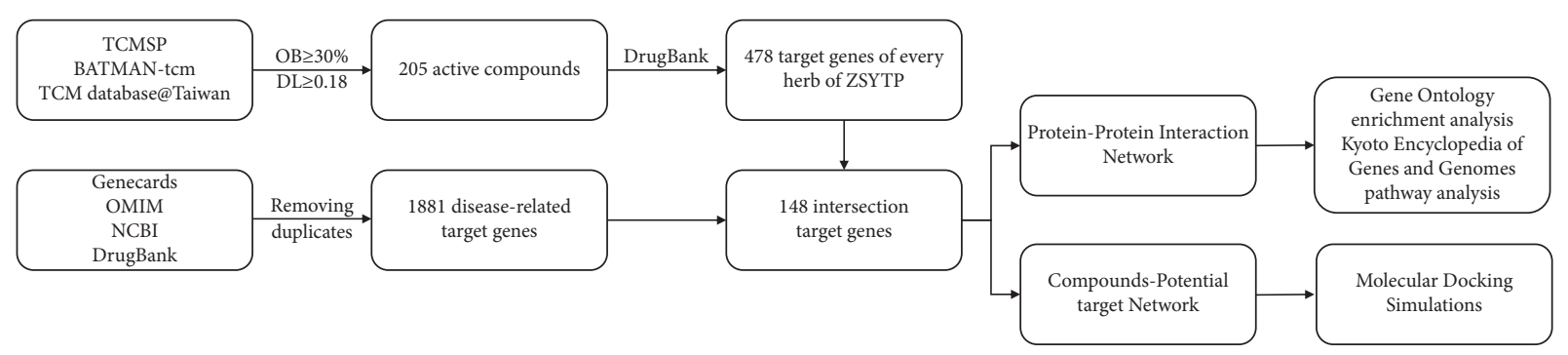

Figure 1: Workflow of the present study.

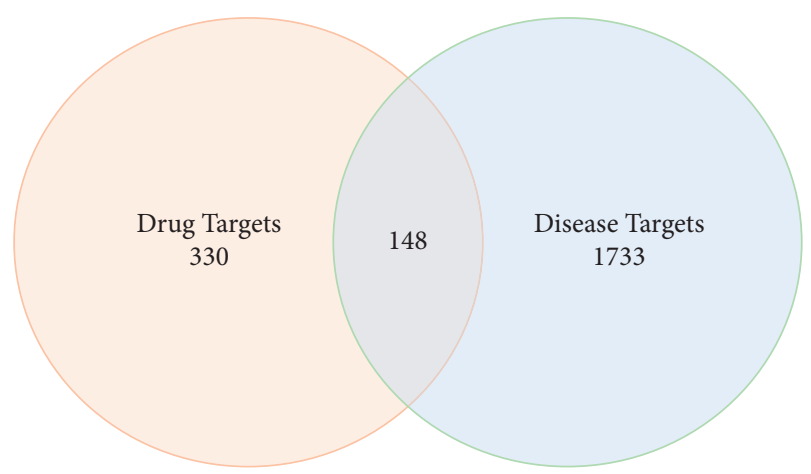

Figure 2: The Venn diagram of intersection potential target genes between drug and disease targets.

regulation of mRNA metabolic process, cellular protein metabolic process, cellular response to stress, intracellular transport, regulation of the nucleobase-containing compound metabolic process, and regulation of nitrogen compound metabolic process.

3.6.2. Gene Ontology Molecular Function (GO-MF). 107 terms were retrieved in gene ontology analysis of MF. Twenty molecular functions with the ascending $p$ values were selected for further analysis and visualized in Figure 5(b). Gene ontology analysis of molecular function highlighted mRNA binding, enzyme binding, and cadherin binding as functions with the decreasing $p$ value.

3.6.3. Gene Ontology Cellular Component (GO-CC). 114 components were identified in gene ontology analysis of CC, including focal adhesion, cytosolic large ribosomal subunit, spliceosomal complex, cytosolic small ribosomal subunit, vesicle, extracellular space, catalytic Step 2 spliceosome, nucleolus, nuclear chromosome, chromosome, anchoring junction, chromosomal region, nuclear body, nuclear speck, polysome, and nuclear periphery. Twenty cellular components with ascending $p$ values were selected for further analysis and visualized in Figure 5(c).

3.6.4. KEGG Pathway Analysis. Further KEGG analysis highlighted 16 pathways of ZSYTP potential pharmacological mechanisms on PCOS. All pathways are presented in Figure 5(d), and further visualization of the KEGG pathway is shown in Figure 6.
3.7. Molecular Docking Visualization. The lowest binding energy of molecular docking of potential targets and their appointed compounds is shown in Table 2 and the information of potential targets is shown in Table 3. In accordance with previous research, the binding activity of molecular docking simulations was considered that it is practicable when the binding energy was lower than $-1.2 \mathrm{kcal} / \mathrm{mol}(-5 \mathrm{~kJ} / \mathrm{mol})$, and it is dynamite when the binding energy was lower than $-5.0 \mathrm{kcal} /$ mol. As presented in Table 2, all of the binding energy between the macromolecule and small-molecule is lower than $-1.2 \mathrm{kcal} /$ mol. $23.3 \%$ of the binding energy is lower than $-5.0 \mathrm{kcal} / \mathrm{mol}$. Furthermore, after molecular docking, the potential targets with their original ligands and the comparison of the lowest binding energy between potential targets-drug compounds and the targets-original ligands showed that the drug compounds were showing potential to be the active compounds of the potential targets in vivo.

The molecular docking simulations of GSK3B helenalin, F2 beta-sitosterol, F2 helenalin, DPP4 helenalin, CYP1B1 beta-sitosterol, and CYP1B1-helenalin are shown in Figures $7-12$, respectively.

These figures show the residues and hydrogen bonds that the ligand binds to the protein. The residues are indicated in bluish violet, while the hydrogen bonds are dotted in yellow.

Visualization of beta-sitosterol in Lycii Fructus, Cuscutae Semen, Eucommiae Cortex, Artemisiae argyi, Panax ginseng, Morindae officinalis Radix, and Amomum aurantiacum and helenalin in Eucommiae Cortex among the disease targets is shown to be stable and feasible. Therefore, these may warrant more research for further validation of the therapeutic effects of ZSYTP.

\section{Discussion}

ZSYTP was first used to prevent recurrent spontaneous and threatened abortion. In line with TCM's theory of treating different diseases with the same therapeutic principle and on the basis that the kidney in TCM's theory is closely related to reproduction, ZSYTP is also used to treat irregular menstruation and infertility in clinical practice, such as PCOS [26-30].

It has been found that ZSYTP can improve the clinical symptoms of PCOS patients [5]. Therefore, our team sought to elucidate ZSYTP's pharmacological mechanism on PCOS.

In order to further support the existing literature and provide insights and possible hypotheses for the pharmacological mechanism of ZSYTP in the treatment of PCOS, we conducted extensive literature research on the active 


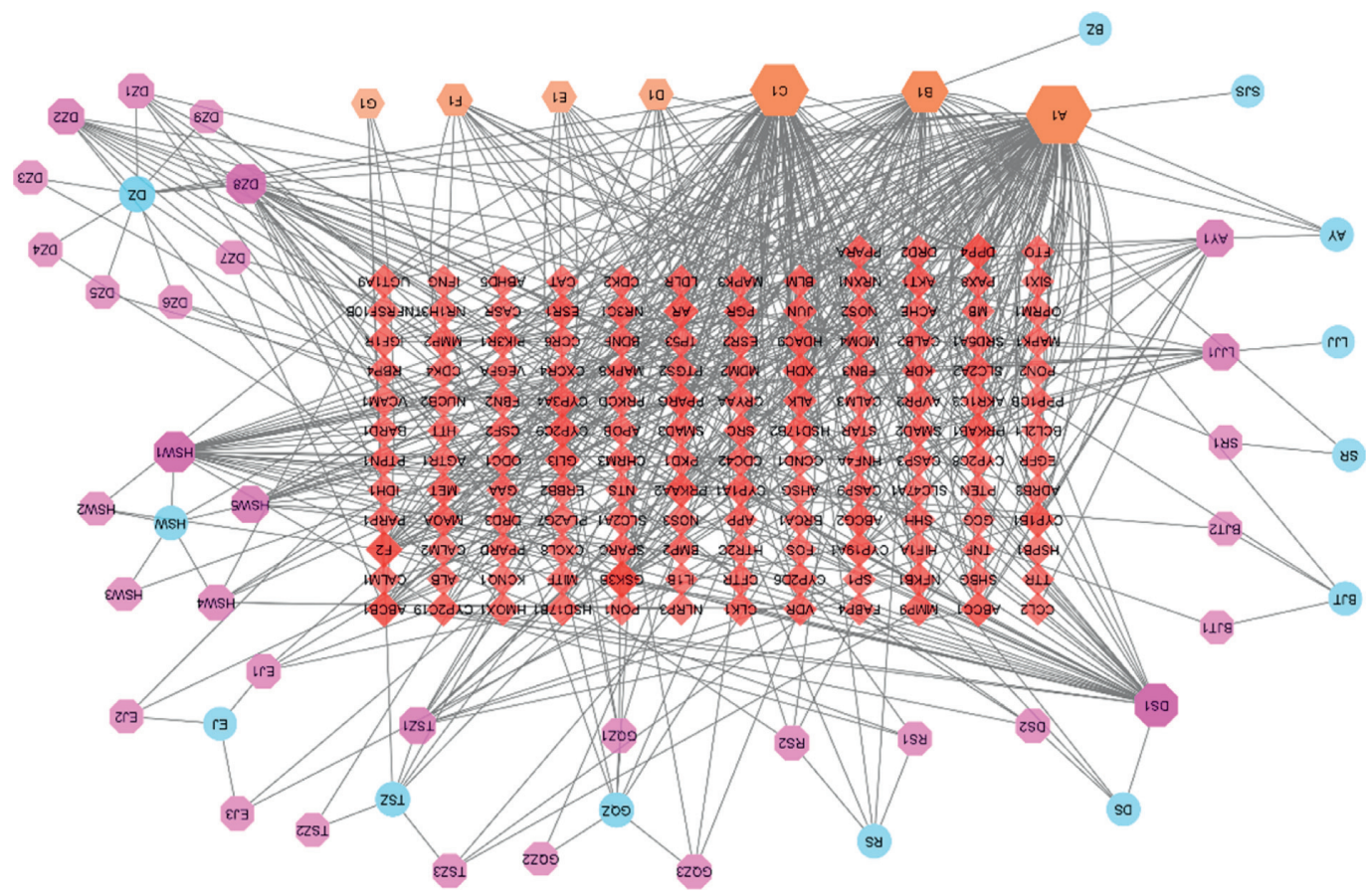

Figure 3: Compound-potential target network. Each blue ellipse represents a herb in ZSYT, and the pink octagons represent active compounds. The red diamonds represent the potential targets, and the orange hexagons at the bottom represent common active compounds. The size and transparency of each node are proportional to the target degree in the network.

TABLE 1: List of top six active compounds of ZSYTP based on degree.

\begin{tabular}{lcc}
\hline Active compound & Degree & Herbs involved \\
\hline Quercetin & 125 & GQZ, TSZ, DZ, AY, and SJS \\
Kaempferol & 96 & TSZ, DZ, and RS \\
Beta-Sitosterol & 54 & GQZ, TSZ, DZ, AY, RS, BJT, SR, HSW, and BZ \\
Luteolin & 35 & DS \\
Emodin & 26 & HSW \\
Helenalin & 23 & DZ \\
\hline
\end{tabular}

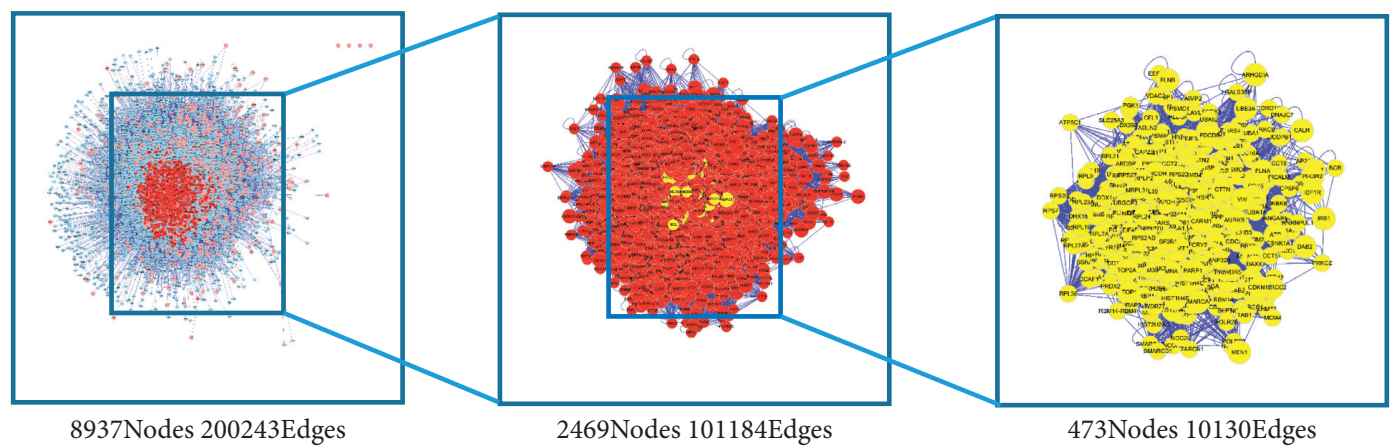

Figure 4: Topological screening process of PPI network.

compounds as highlighted by the PPI networks and 16 pathways as indicated by KEGG pathway enrichment analysis.

4.1. Regulation of HIF-1 Expression Levels and Ovulation. As a tonifying kidney TCM formula, ZSYTP is found to have therapeutic effects in the treatment of POI and PCOS [31].
However, the pharmacological mechanisms of ZSYTP remain unclear.

Our team conducted a network pharmacology study on the effect of ZSYTP on POI. One hypothesis put forward by our previous study was that ZSYTP upregulates the hypoxiainducible factor-1 (HIF1) signaling pathway [32], thereby encouraging oocyte competence through a multitude of pathways. Similarly, the KEGG analysis of ZSYTP on PCOS 


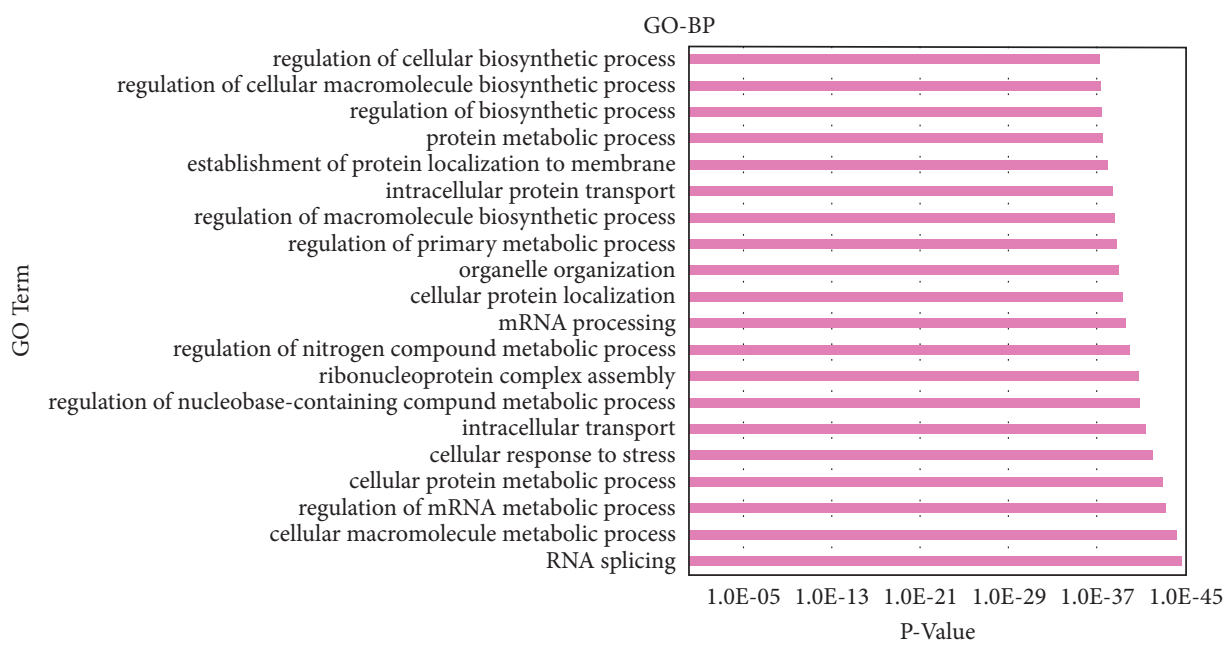

(a)

GO-MF

RNA polymerase II-specific DNA-binding transcription factor... heat shock protein bindin DNA-dependent ATPase activity protein kinase binding DNA-binding transcription factor binding adenyl nucleotide binding ubiquitin protein ligase bindin ATP binding ribonucleotide binding purine nucleotide bindin purine ribonucleoside triphosphate binding nucleoside phosphate binding transcription factor binding cell adhesion molecule bindin ATPase activity
cadherin binding cadherin binding mRNA binding

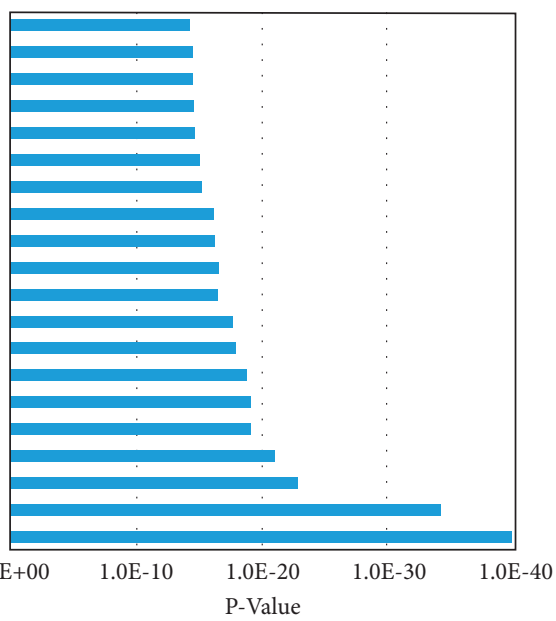

(b)

GO-KEGG

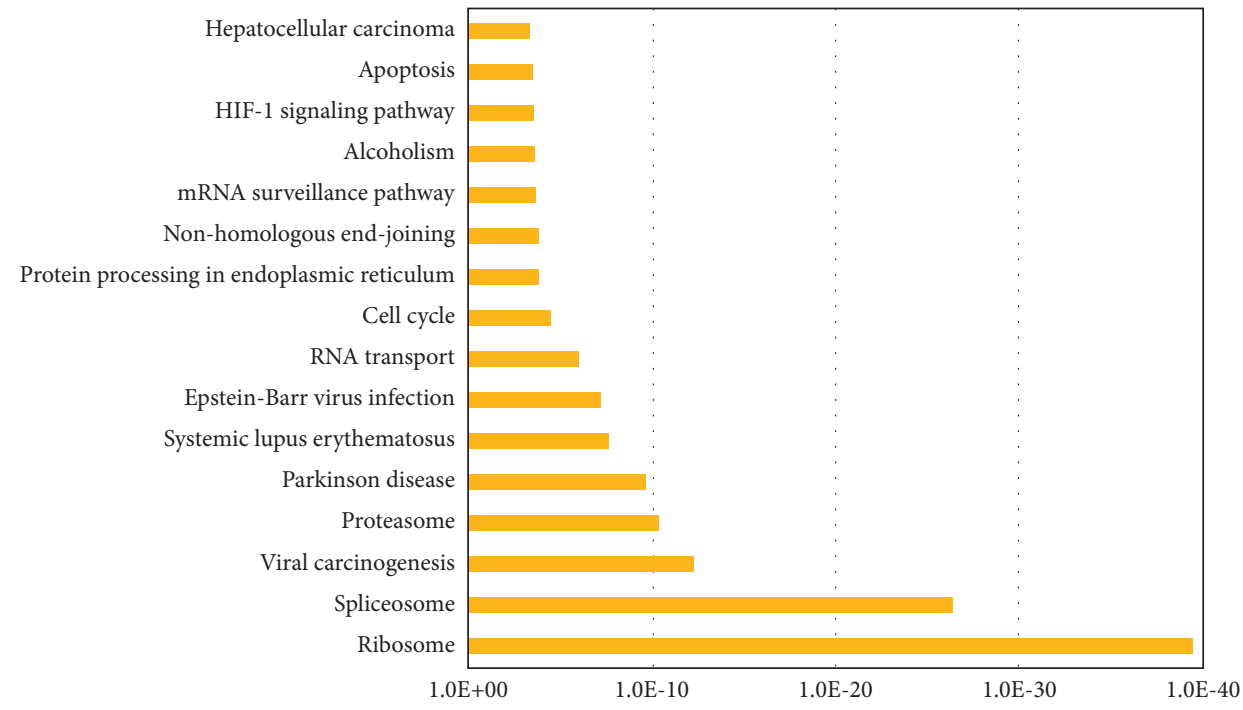

(c)

FiguRe 5: Continued. 


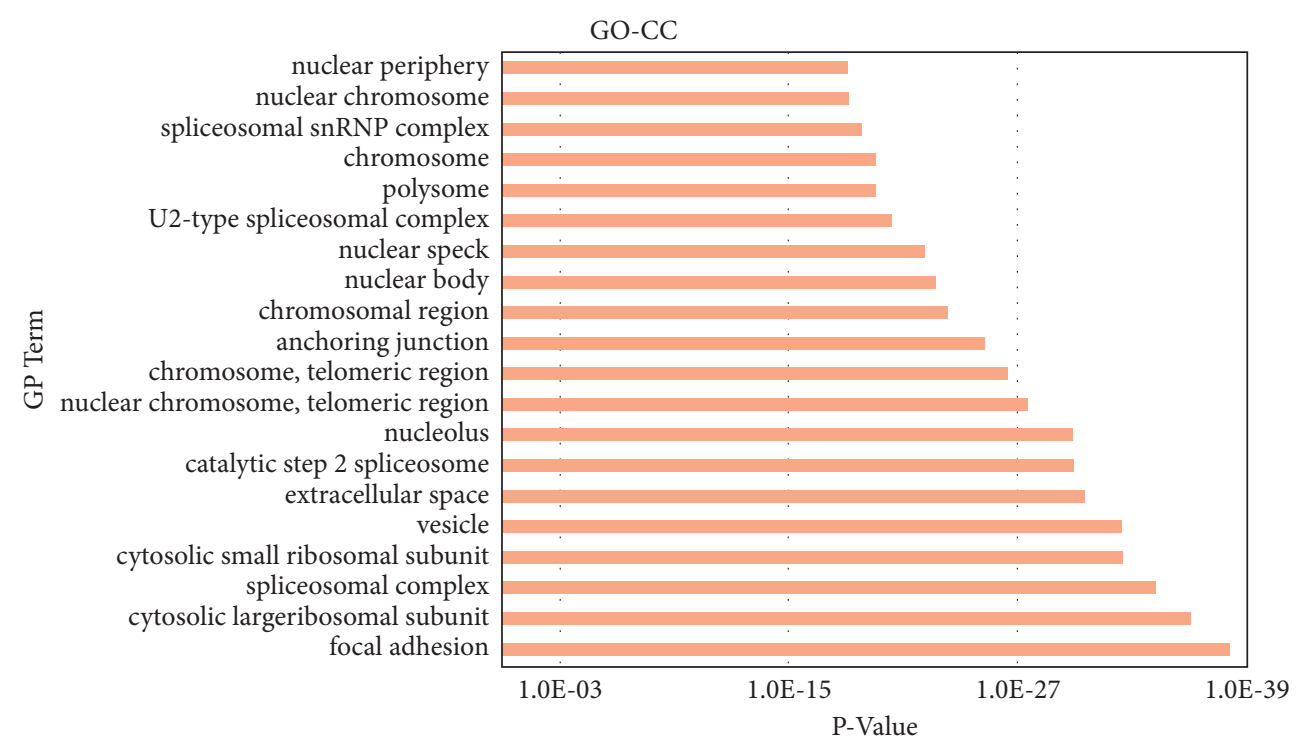

(d)

FIgURE 5: The GO function and KEGG pathway enrichments. (a) Enriched BP functions of active target genes. (b) Enriched MF functions of active target genes. (c) Enriched CC functions of active target genes. (d) KEGG pathway enrichment. BP: biological process; MF: molecular function; CC: cellular component; KEGG: Kyoto Encyclopedia of Genes and Genomes.

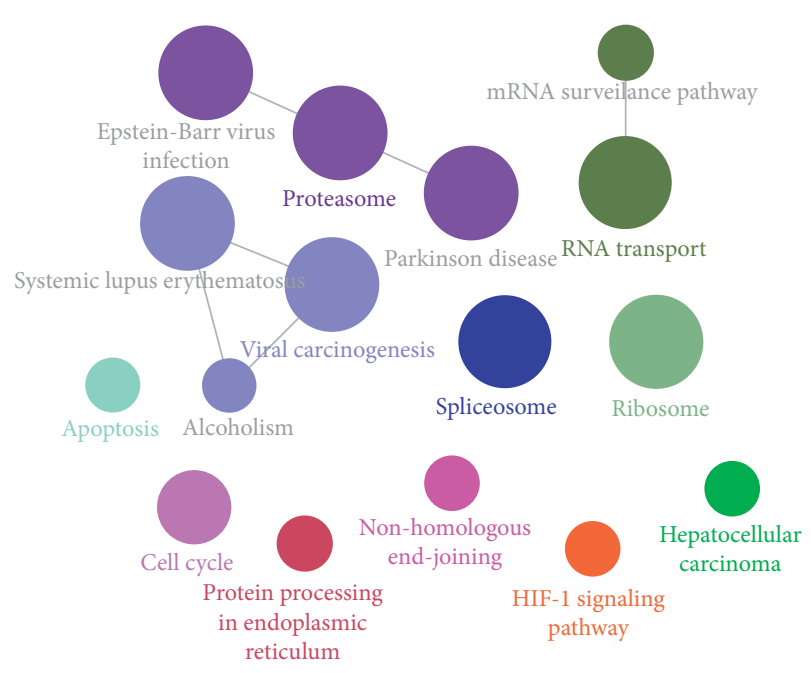

FIGURE 6: KEGG pathway enrichment analysis visualized by ClueGO.

highlighted the HIF-1 pathway, indicating a possible relationship between the treatment efficacy of ZSYTP and this pathway.

HIF-1 is a transcription factor, and its activity is mainly determined by HIF- $1 \alpha$, which is regulated by the low oxygen concentration of the environment [33]. Human oocytes grow in follicular fluid surrounded by a basement membrane, where there is a lack of blood supply [34] and a low oxygen concentration of about $1.3 \%-5.5 \%$ in the human follicular fluid [35]. Development of the follicle, subsequent maturation, and ovulation all through to the early luteal phase takes place in a hypoxic environment [36].
One study showed that polycystic ovaries were found in rats when submitted into hypobaric hypoxia. Weight of the ovaries increased under a hypoxic environment, while synthesis and secretion of E2 were negligible [37].

Multiple animal experimental studies suggest that HIF-1 promotes follicular development and interacts with FSH receptors on granulosa cells to regulate E2 secretion [38]. One other study found high expressions of HIF-1 mRNA in dominant follicles and negligible amounts of HIF-1 mRNA expression in preatretic follicles, suggesting that HIF-1 expression might be crucial in follicular development [39]. This is further substantiated by another study investigating the expression patterns of HIF-1a in endometrial tissues of PCOS and healthy women. The study found that mRNA and HIF- $1 \alpha$ protein expression in PCOS women were significantly lower than those of the control group, indicating HIF$1 \alpha^{\prime}$ s possible involvement in the molecular mechanisms of endometrial dysfunction in PCOS women, affecting fertility [40].

The balance between HIF-1 expression and oxygen concentration of the environment is therefore crucial for normal follicle development and subsequent ovulation to take place.

Quercetin is an active compound in ZSYTP with the highest degree, as indicated in the Compound-Potential Target Network (Figure 3). It has been widely reported that hypoxia is the only known naturally occurring signal to activate the HIF-1 transcription factor. A recent study concluded that quercetin activates HIF- $1 \alpha$ in a manner similar to hypoxia and stabilizes HIF-1 $\alpha$ [41]. Quercetin is also found to reduce high testosterone levels, luteinizing hormone (LH), and insulin resistance in PCOS. Multiple studies have postulated that the benefits of quercetin could be due to its antioxidant and anti-inflammatory properties 
TABLE 2: Lowest binding energy of compounds-target molecular docking.

\begin{tabular}{lcccccccc}
\hline Target & Degree value & Original ligands & Quercetin & Kaempferol & Beta-Sitosterol & Luteolin & Emodin & Helenalin \\
\hline GSK3B & 19 & -3.61 & -1.86 & -2.5 & -4.31 & -2.37 & -1.53 & -5.02 \\
F2 & 15 & -2.06 & -2.97 & -4.82 & -6.67 & -4.48 & -4.62 & -6.95 \\
ABCB1 & 13 & -2.33 & -1.76 & -2.28 & -4.14 & -2.04 & -3.57 & -4.57 \\
DPP4 & 13 & -2.19 & -2.51 & -2.64 & -4.36 & -2.39 & -3.97 & -5.72 \\
CYP1B1 & 12 & -6.43 & -2.99 & -3.76 & -5.72 & -4.96 & -4.6 & -6.33 \\
\hline
\end{tabular}

GSK3B: glycogen synthase kinase 3 beta; F2: coagulation factor II; ABCB1: ATP binding cassette subfamily B member 1; DPP4: dipeptidyl peptidase 4; CYP1B1: cytochrome P450 1B1.

TABLE 3: The information of potential targets.

\begin{tabular}{lcc}
\hline Protein name & Gene name & PDB ID \\
\hline Glycogen synthase kinase 3 beta & GSK3B & 3F88 \\
Coagulation factor II, thrombin & F2 & 2AFQ \\
ATP binding cassette subfamily B member 1 & ABCB1 & 7A69 \\
Dipeptidyl peptidase 4 & DPP4 & 6B1E \\
Cytochrome P450 1B1 & CYP1B1 & 3PM0 \\
\hline
\end{tabular}

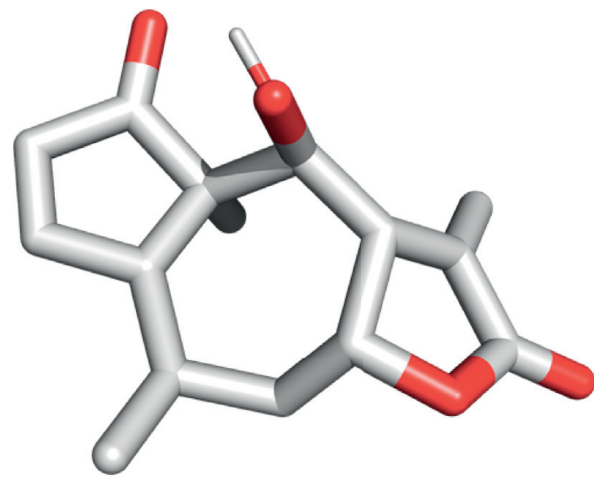

(a)

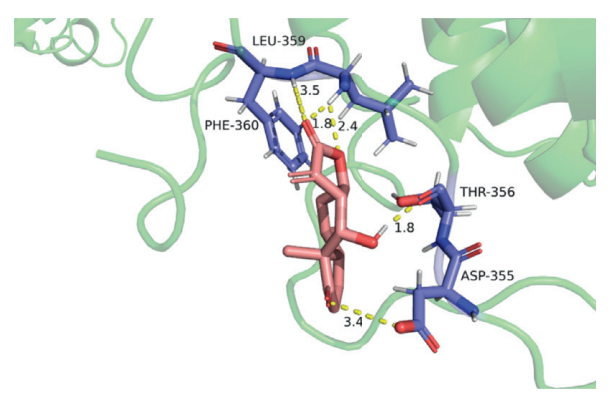

(c)
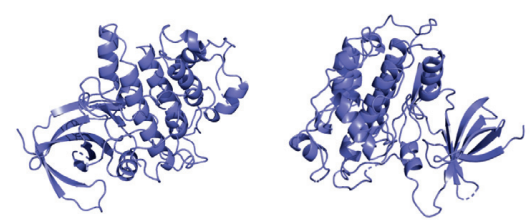

(b)
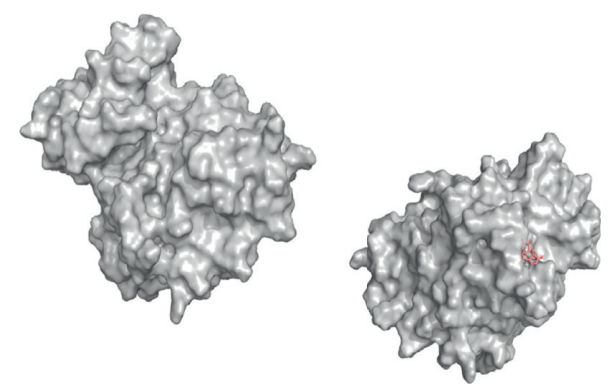

(d)

FIGURE 7: GSK3B helenalin molecular docking. 3D structures of (a) helenalin and (b) GSK3B. (c) Molecular docking simulation and (d) display protein surface of molecular docking simulation.

and concluded that quercetin could be considered as a possible treatment for PCOS [42].

It is possible that the active compounds in ZSYTP like quercetin activate the HIF-1 transcription factor, promoting follicular development in PCOS patients and thereby reducing follicular atresia and regulating ovulation.

4.2. Insulin Resistance and Ovarian Function. PCOS women have an increased risk of insulin resistance (IR), while multiple studies have shown that IR plays an important role in the occurrence and development of PCOS as well [43]. Due to impaired use of glucose by peripheral tissues, the pancreas secretes more insulin in response to the increased glucose levels in the blood. This process involves the activation of the phosphatidylinositol-3-phosphate kinase (PI3K) and protein kinase B (AKT) pathway [44].

When PI3K is activated, it affects the intracellular glucose transporter (GLUT) to transport glucose across the membrane. Studies have shown that GLUT4 expression in 


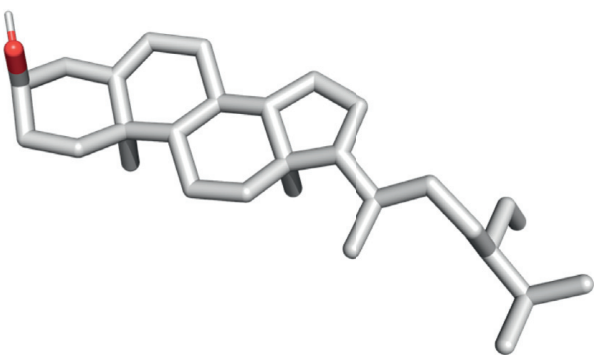

(a)

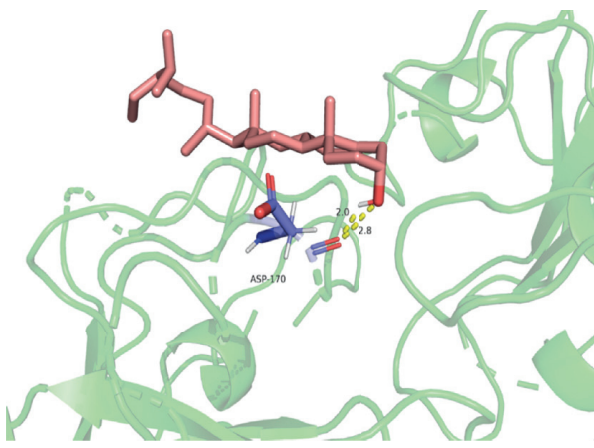

(c)

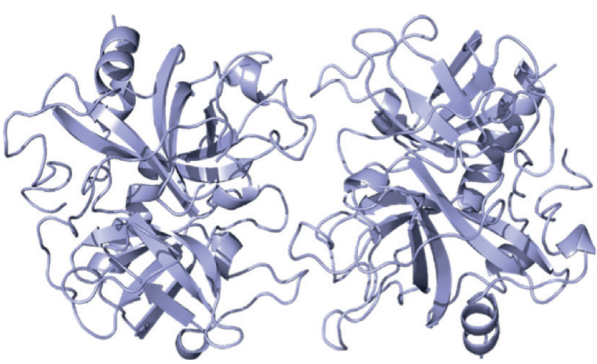

(b)

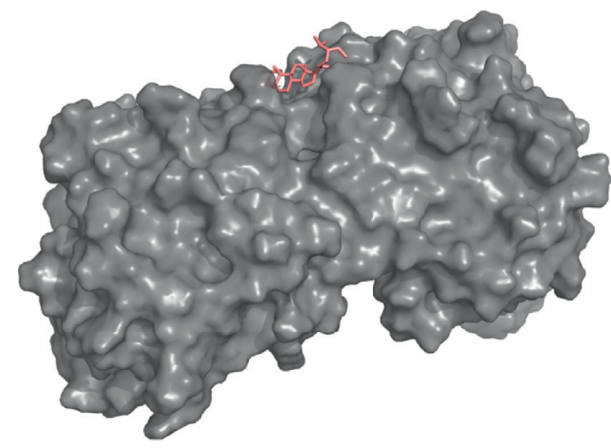

(d)

Figure 8: F2 beta-sitosterol molecular docking. 3D structures of (a) beta-sitosterol and (b) F2. (c) Molecular docking simulation and (d) display protein surface of molecular docking simulation.

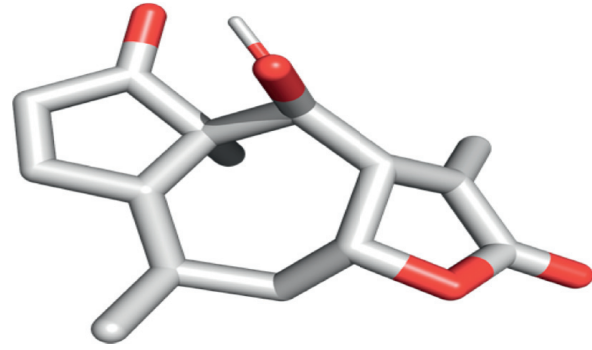

(a)

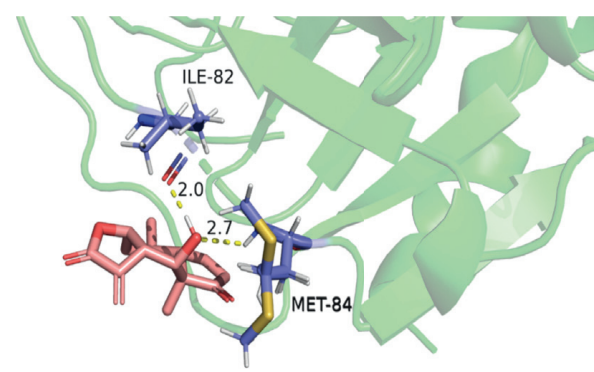

(c)

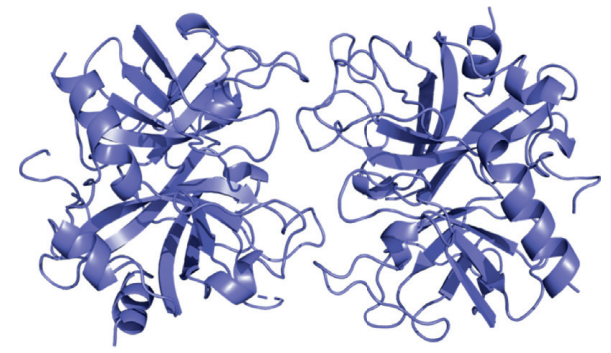

(b)

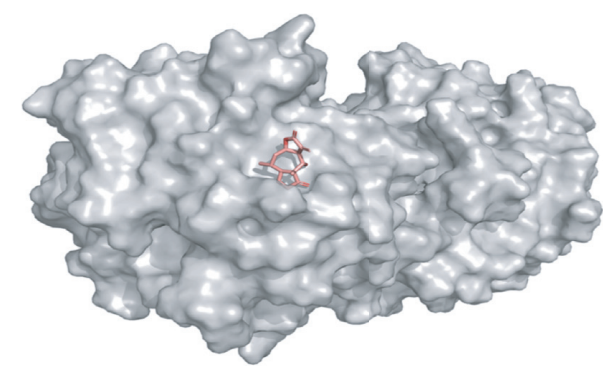

(d)

Figure 9: F2 helenalin molecular docking. 3D structures of (a) helenalin and (b) F2. (c) Molecular docking simulation and (d) display protein surface of molecular docking simulation.

the PCOS uterus is significantly reduced, thus affecting glucose metabolism in the body. Quercetin, the active ingredient in ZSYTP, can increase the expression of this gene [45]. In addition to improving ovarian function, it can also improve endometrial function and inflammatory microenvironment by regulating IR state [46]. At the same time, androgen secretion can be decreased by downregulating the expression of the CYP17A1 gene [47].

Normally, all female follicles develop through a series of processes such as recruitment, selection, dominance, and ovulation, in which physiological concentrations of insulin levels promote granulosa cell proliferation, and glucose 


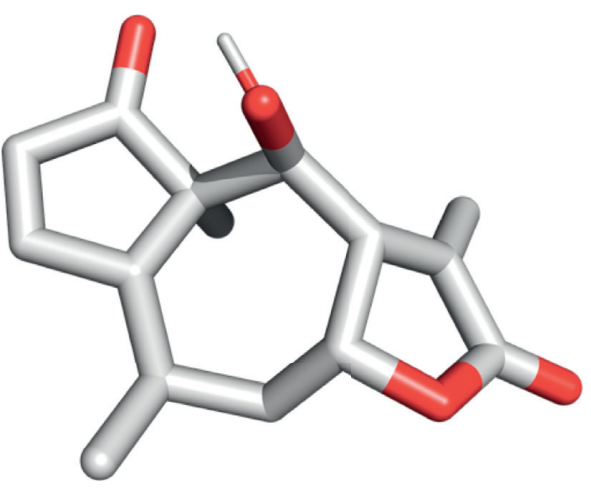

(a)

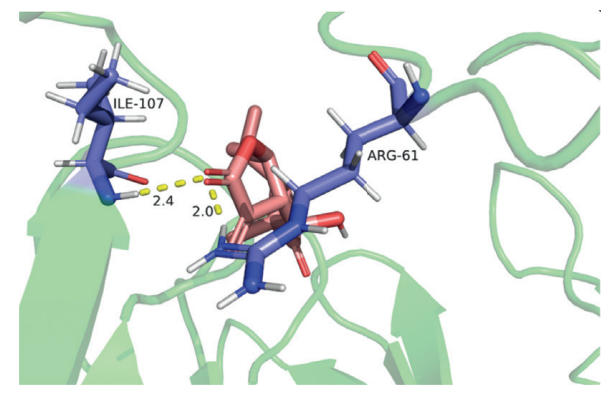

(c)

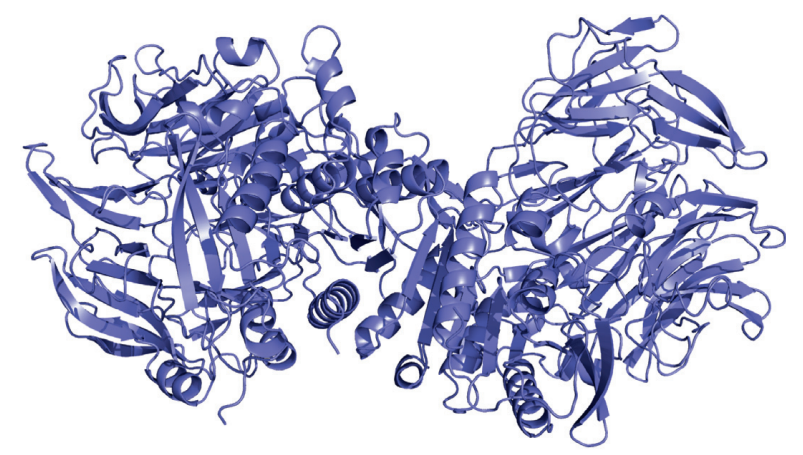

(b)

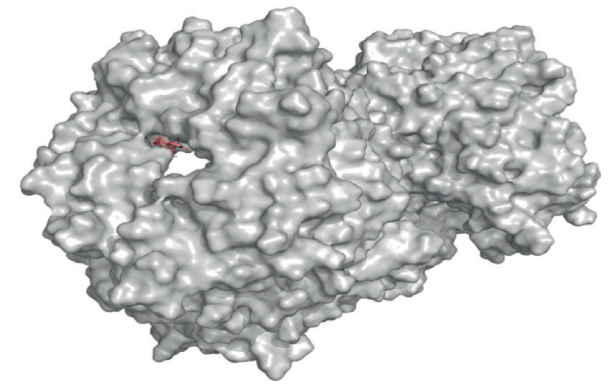

(d)

FIGURE 10: DPP4 helenalin molecular docking. 3D structures of (a) helenalin and (b) DPP4. (c) Molecular docking simulation and (d) display protein surface of molecular docking simulation.

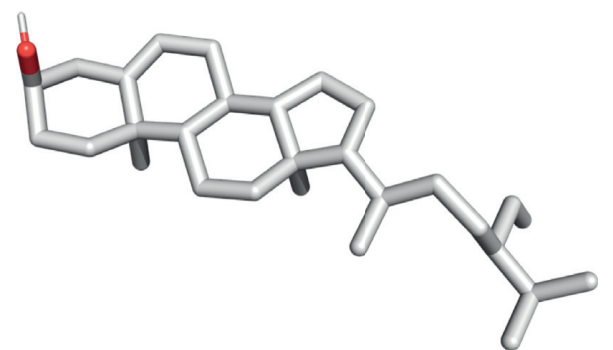

(a)

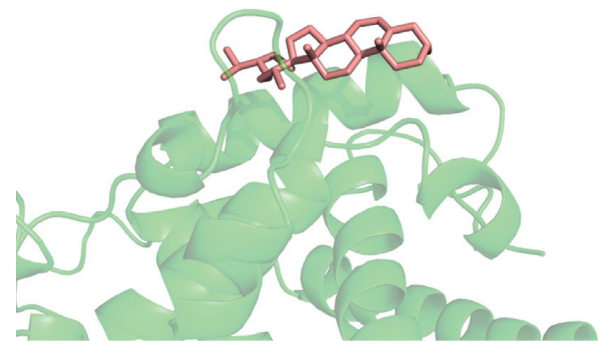

(c)

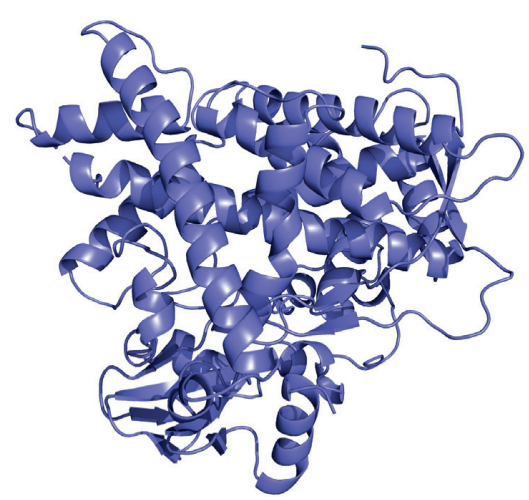

(b)

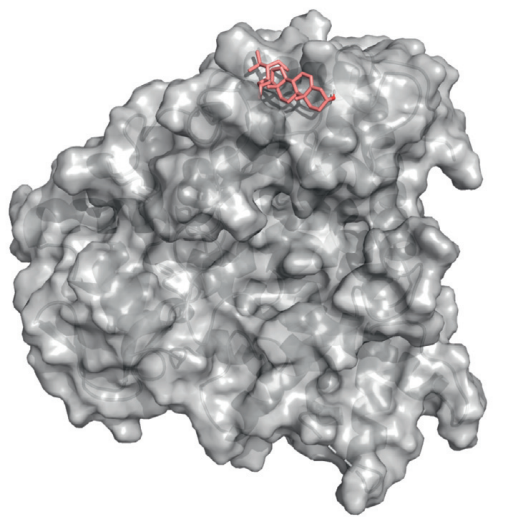

(d)

Figure 11: CYP1B1 beta-sitosterol molecular docking. 3D structures of (a) beta-sitosterol and (b) CYP1B1. (c) Molecular docking simulation and $(\mathrm{d})$ display protein surface of molecular docking simulation. 


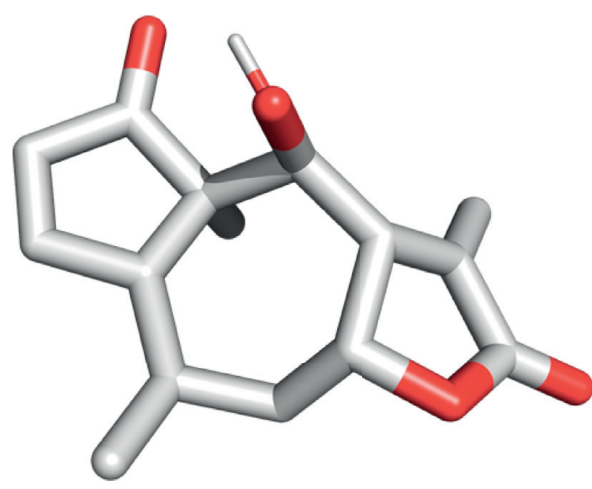

(a)

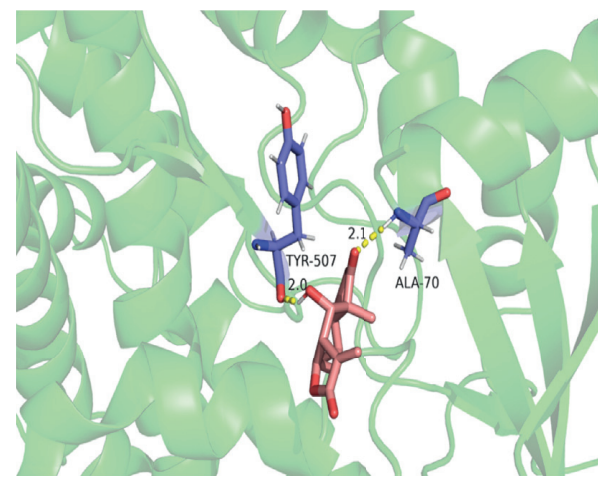

(c)

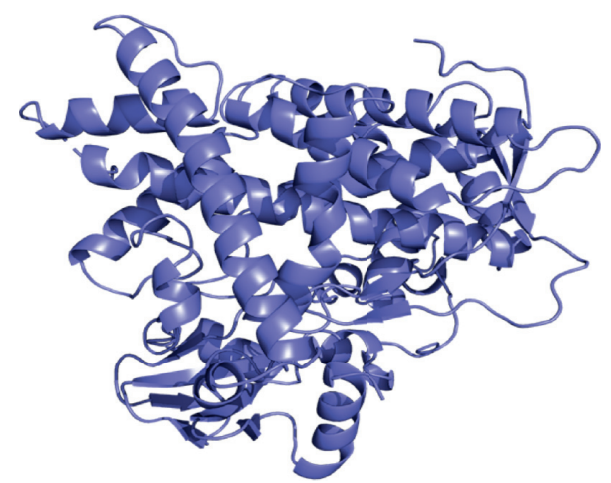

(b)

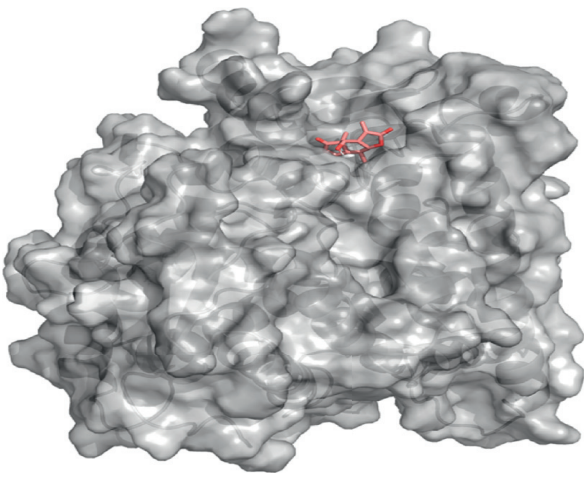

(d)

FIGURE 12: CYP1B1 helenalin molecular docking. 3D structures of (a) helenalin and (b) 3D structures of CYP1B1. (c) Molecular docking simulation and $(\mathrm{d})$ display protein surface of molecular docking simulation.

metabolism in granulosa cells provides energy for oocyte development, thereby regulating follicular development. In addition, the insulin signaling pathway affects ovarian physiological function, oocyte developmental quality, and the ovulation process [48].

In the PPI network, through the analysis of the active components and targets of ZSYTP, it is predicted that the main active components and targets of ZSYTP have a good potential to improve IR in PCOS. Not only quercetin but also luteolin and emodin have a multidirectional regulation effect on the etiology of PCOS that has been explored [49].

Luteolin and emodin can improve the environment of high insulin levels by promoting PI3K/AKT pathway $[50,51]$ and thus upregulating CYP1B1 gene expression, which is a potential target gene of ZSYTP and PCOS, and CYP1B1 is involved in steroid and lipogenesis and metabolism [52] and also relates to improving the aromatase activity of ovarian granulosa cells in an environment of high insulin levels, which enhanced expression of FSH receptors and increased estrogen produciton. The sensitivity of granulosa cells to FSH was increased, thereby improving follicular dysgenesis [53], which in turn reduces the number of atretic follicles and restores the motility cycle.

Dipeptidyl peptidase-4 (DPP4) is one of the main potential target genes of ZSYTP and PCOS, and studies have shown that it plays a key role in the regulation of insulin secretion. DPP4 serum activity is elevated in patients with polycystic ovary syndrome, DPP4 serum levels are closely related to anti-Müllerian hormone (AMH) serum levels [54], and the use of DPP4 inhibitors may improve IR [55].
Patients with PCOS often have symptoms of ovulatory disturbance or anovulation, which may be related to their IR, and our results from molecular docking suggest that ZSYTP may improve IR associated with PCOS, thus directly or indirectly improving ovarian function.

4.3. Relation between Epigenetics and PCOS. Gene expression in eukaryotes is performed by RNA polymerase, spliceosome, and ribosome, respectively [56], and KEGG pathway enrichment analysis in the study shows that the mechanism of ZSYTP in treating PCOS was closely related to these dynamic molecular machines. PCOS patients have the characteristics of family aggregation and heterogeneity [57], which also suggests that PCOS is closely related to the combined effects of genetics and environment. An abnormal epigenetic modification is closely related to the occurrence and inheritance of multiple diseases, which may be the medium between environmental factors and human diseases [58]. Moreover, with different changes in the gene nucleotide sequence, epigenetic changes are often reversible [59], which provides a new thought for the mechanism study of ZSYTP in the treatment of PCOS.

\section{Conclusion}

The pathogenesis of PCOS still remains undefined; therefore, there is no effective cure for this disease. Although most fertility problems in PCOS patients can be resolved through 
symptomatic treatment, PCOS still severely affects women's long-term health.

Hence, this paper aims to investigate the possible etiology of PCOS and explore the pharmacological mechanism between ZSYTP and PCOS. We extracted 205 active compounds from ZSYTP and simultaneously retrieved 1881 disease target genes on multiple databases. 148 intersection target genes were derived by intersecting the databases of disease and drug-target genes.

Subsequently, our team constructed C-T network and multiple PPI networks and performed the GO and KEGG analysis. This study hypothesizes that ZSYTP may alleviate IR to treat PCOS. GO and KEGG analysis also highlighted that the HIF-1 signaling pathway and gene expression could be the pathological mechanisms of PCOS.

Additionally, the result of this research shows that the same compounds in ZSYTP could regulate multiple targets; meanwhile, the same targets could intervene in multiple biological processes and pathways.

It reflects the characteristics of the combined action of multipathway and multitarget of ZSYTP. The results suggested that the whole network can be regulated by regulating single or multiple targets in the network, which provides a scientific basis and new insights for the clinical application of ZSYTP in the treatment of PCOS and simultaneously provides a new direction for further exploring the potential mechanism of ZSYTP on PCOS.

This study is mainly based on bioinformatics and massive data calculation results, which will be further screened and verified by animal or cell experiments to clarify the main regulatory targets of ZSYTP, thus promoting the development of modern pharmacology of TCM.

\section{Abbreviations}

$\begin{array}{ll}\text { RS: } & \text { Panax ginseng } \\ \text { GQZ: } & \text { Lycii Fructus } \\ \text { BJT: } & \text { Morindae officinalis Radix } \\ \text { SR: } & \text { Amomum aurantiacum } \\ \text { AY: } & \text { Folium artemisiae Argyi } \\ \text { DS: } & \text { Codonopsis Radix } \\ \text { DZ: } & \text { Eucommiae Cortex } \\ \text { SDH: } & \text { Rehmanniae Radix Praeparata } \\ \text { BZ: } & \text { Atractylodes macrocephala Koidz } \\ \text { SJS: } & \text { Herba Taxilli } \\ \text { XD: } & \text { Dipsaci Radix } \\ \text { TSZ: } & \text { Cuscutae Semen } \\ \text { HSW: } & \text { Fallopia multiflora } \\ \text { EJ: } & \text { Colla Corii Asini } \\ \text { LJS: } & \text { Cornu Cervi Degelatinum } \\ \text { OB: } & \text { Oral bioavailability } \\ \text { DL: } & \text { Drug-likeness } \\ \text { TCM: } & \text { Traditional Chinese medicine } \\ \text { ZSYTP: } & \text { Zishen Yutai Pills } \\ \text { PCOS: } & \text { Polycystic ovary syndrome } \\ \text { ADME: } & \text { Adsorption, Distribution, Metabolism, Excretion } \\ \text { HIF-1: } & \text { Hypoxia-inducible factor 1 } \\ \text { IR: } & \text { Insulin resistance. }\end{array}$

\section{Data Availability}

The datasets supporting the conclusions of this article are available in a public database from TCMSP, TCM Database@ Taiwan, BATMAN-TCM, Swiss Target Prediction, STITCH, PubChem, DrugBank, UniProt, GeneCards, OMIM, NCBI, and RCSB PDB.

\section{Conflicts of Interest}

The authors declare that they have no conflicts of interest.

\section{Authors' Contributions}

Ying Zhao and Yihui Feng formulated the idea of the paper and supervised the research. Yingyin Chen performed the research, analyzed the data, and wrote the paper. Xinyi Chai participated in revising the data and improving paper writing. Xinqian Yang and Caiting Zhong made a contribution to the part of target database building, data analysis, and interpretation. Yihui Feng and Ying Zhao supervised the study and conducted the revision of the paper. All authors reviewed the paper and read and approved the final version of the paper.

\section{Acknowledgments}

The study was supported by the National Chinese Medicine Academic Schools Inherit Workstation Project ([2019]62), the Zishen Yutai Pills Scientific Fund Project for Young and Middle-Age Researchers supported by Guangzhou Baiyunshan Zhongyi Pharmaceutical Co., Ltd (20190803), and The Program of "Hundred-Thousand-Ten Thousand" of Inheritance and Innovation of Traditional Chinese Medicine ([2018]284).

\section{References}

[1] R. Azziz, "Introduction: determinants of polycystic ovary syndrome," Fertility and Sterility, vol. 106, no. 1, pp. 4-5, 2016.

[2] L. G. Cooney and A. Dokras, "Beyond fertility: polycystic ovary syndrome and long-term health," Fertility and Sterility, vol. 110, no. 5, pp. 794-809, 2018.

[3] D. Lizneva, L. Suturina, W. Walker, S. Brakta, L. GavrilovaJordan, and R. Azziz, "Criteria, prevalence, and phenotypes of polycystic ovary syndrome," Fertility and Sterility, vol. 106, no. 1, pp. 6-15, 2016.

[4] Z. Chen, R. Chen, J. Chen et al., "China's diagnosis and treatment guidelines of polycystic ovary syndrome," Chinese Journal of Obstetrics and Gynecology, vol. 53, no. 1, pp. 2-6, 2018.

[5] Y. Zhao, L. Cao, and S. Luo, "Clinical research and applications of zishen Yutai pill," World Journal of Traditional Chinese Medicine (WJTCM), vol. 6, no. 4, pp. 318-319, 2011.

[6] Y. Zheng, "An interventional research of zishen Yutai Pills' ovulatory effect on kidney deficient PCOS patients," Journal of Guangzhou University of Traditional Chinese Medicine, vol. 53, no. 1, pp. 2-6, 2018.

[7] H. Yuan, Q. Ma, H. Cui et al., "How can synergism of traditional medicines benefit from network pharmacology?" Molecules, vol. 22, no. 7, p. 1135, 2017. 
[8] Y. Tu, "The discovery of artemisinin (Qinghaosu) and gifts from Chinese medicine," Nature Medicine, vol. 17, no. 10, pp. 1217-1220, 2011.

[9] J. Ru, P. Li, J. Wang et al., "TCMSP: a database of systems pharmacology for drug discovery from herbal medicines," Journal of Cheminformatics, vol. 6, no. 1, p. 13, 2014.

[10] Z. Liu, F. Guo, and Y. Wang, "BATMAN-TCM: a bioinformatics analysis tool for molecular mechanism of traditional Chinese medicine," Scientific Reports, vol. 6, no. 1, Article ID 21146, 2016.

[11] C. Y.-C. Chen, “TCM Database@Taiwan: the world's largest traditional Chinese medicine database for drug screening in silico," PLoS One, vol. 6, no. 1, Article ID e15939, 2011.

[12] S. Kim, J. Chen, T. Cheng et al., "PubChem in 2021: new data content and improved web interfaces," Nucleic Acids Research, vol. 49, no. D1, pp. D1388-D1395, 2021.

[13] D. Szklarczyk, A. Santos, and C. Von Mering, "Stitch 5: augmenting protein-chemical interaction networks with tissue and affinity data," Nucleic Acids Research, vol. 44, no. D1, pp. D380-D384, 2016.

[14] A. Daina, O. Michielin, and V. Zoete, "SwissTargetPrediction: updated data and new features for efficient prediction of protein targets of small molecules," Nucleic Acids Research, vol. 47, no. W1, pp. W357-W364, 2019.

[15] D. S. Wishart, Y. D. Feunang, and A. C. Guo, "DrugBank 5.0: a major update to the DrugBank database for 2018," Nucleic Acids Research, vol. 46, no. D1, pp. D1074-D1082, 2018.

[16] G. Stelzer, N. Rosen, I. Plaschkes et al., "The GeneCards suite: from gene data mining to disease genome sequence analyses," Current Protocols in Bioinformatics, vol. 54, pp. 1-33, 2016.

[17] J. S. Amberger and A. Hamosh, "Searching online mendelian inheritance in man (OMIM): a knowledgebase of human genes and genetic phenotypes," Current Protocols in Bioinformatics, vol. 58, no. 1, 2017.

[18] E. W. Sayers, R. Agarwala, and E. E. Bolton, "Database resources of the national center for Biotechnology information," Nucleic Acids Research, vol. 47, no. D1, pp. D23-D28, 2019.

[19] U. P. Consortium, "UniProt: the universal protein knowledgebase in 2021," Nucleic Acids Research, vol. 49, no. D1, pp. D480-D489, 2021.

[20] A. Martin, M. E. Ochagavia, and L. C. Rabasa, "BisoGenet: a new tool for gene network building, visualization and analysis,” BMC Bioinformatics, vol. 11, no. 1, p. 91, 2010.

[21] G. Bindea, B. Mlecnik, H. Hackl et al., "ClueGO: a Cytoscape plug-in to decipher functionally grouped gene ontology and pathway annotation networks," Bioinformatics, vol. 25, no. 8, pp. 1091-1093, 2009.

[22] Y. Rose, J. M. Duarte, R. Lowe et al., "RCSB protein data bank: architectural advances towards integrated searching and efficient access to macromolecular structure data from the PDB archive," Journal of Molecular Biology, vol. 433, no. 11, Article ID 166704, 2021.

[23] D. Seeliger and B. L. De Groot, "Ligand docking and binding site analysis with PyMOL and Autodock/Vina," Journal of Computer-Aided Molecular Design, vol. 24, no. 5, pp. 417-422, 2010 .

[24] N. M. O’Boyle, M. Banck, C. A. James, C. Morley, T. Vandermeersch, and G. R. Hutchison, "Open Babel: an open chemical toolbox," Journal of Cheminformatics, vol. 3, no. 1, p. 33, 2011.

[25] G. M. Morris, R. Huey, W. Lindstrom et al., "AutoDock4 and AutoDockTools4: automated docking with selective receptor flexibility," Journal of Computational Chemistry, vol. 30, no. 16, pp. 2785-2791, 2009.
[26] X. Tan, S. Ha, and L. Sun, "Effect of Zishen Yutai pill on ovulation induction in infertile patients with kidney deficiency type of PCOS," Chinese Practical Medicine, vol. 16, no. 5, pp. 143-145, 2021.

[27] K. Li, X. Chen, L. Li, B. Wu, and H. Zhou, "Influence of Zishenyutai pill combined with letrozole for treating women with polycystic ovary syndrome on their levels of E2, and VEGF," Chinese Journal of Family Planning, vol. 29, no. 3, pp. 556-559, 2021.

[28] Y. Li, Y. Wang, and D. Xu, “Therapeutic effect of Zishen Yutai pill on infertility patients with polycystic ovary syndrome and the influence of hormone level," Journal of Contemporary Medicine, vol. 25, no. 8, pp. 48-50, 2019.

[29] Y. Zheng, "The study of Zishen yutai Pill on intervention of kidney deficiency type PCOS patients with infertility ovulation," Guangzhou University of Chinese Medicine, 2016.

[30] H. Xu and U. Luo, "Application of Zishen yutai Pill in ovulation promotion treatment protocols of polycystic ovary syndrome," Chinese Journal of Information on TCM, vol. 15, no. 7, 68 pages, 2008.

[31] K. Maharajan, Q. Xia, X. Duan, P. Tu, Y. Zhang, and K. Liu, "Therapeutic importance of Zishen yutai Pill on the female reproductive health: a review," Journal of Ethnopharmacology, vol. 281, pp. 114523-114613, 2021.

[32] Y. Feng, X. Chai, Y. Chen, Y. Ning, and Y. Zhao, "Network pharmacology approach for predicting targets of zishen Yutai Pills on premature ovarian insufficiency," Evidence-based Complementary and Alternative Medicine, vol. 2021, Article ID 8215454, 15 pages, 2021.

[33] K. M. Du, G. Q. Chen, and Z. Chen, "Regulation of hypoxiainducible factor-1 alpha expression," Ai Zheng, vol. 23, no. 9, pp. 1098-1102, 2004.

[34] K. K. Tam, D. L Russell, D. J Peet et al., "Hormonally regulated follicle differentiation and luteinization in the mouse is associated with hypoxia inducible factor activity," Molecular and Cellular Endocrinology, vol. 327, no. 1-2, pp. 47-55, 2010.

[35] A. P. A. Van Montfoort, E. G. J. M. Arts, L. Wijnandts et al., "Reduced oxygen concentration during human IVF culture improves embryo utilization and cumulative pregnancy rates per cycle," Human Reproduction Open, vol. 2020, Article ID hoz036, 2020.

[36] F. Yang, B. Y. Zhang, G. D. Feng et al., "Molecular mechanisms of ovarian hypoxic microenviroment regulating ovulation in mammals," Hereditas, vol. 38, no. 2, pp. 109-117, 2016.

[37] I. H. Martin and L. E. Costa, "Reproductive function in female rats submitted to chronic hypobaric hypoxia," Archives Internationales de Physiologie, de Biochimie et de Biophysique, vol. 100, no. 5, pp. 327-330, 1992.

[38] U. Boonyaprakob, J. E. Gadsby, V. Hedgpeth, P. A. Routh, and G. W. Almondm, "Expression and localization of hypoxia inducible factor-l alpha mRNA in the porcine ovary," $\mathrm{Ca}$ nadian Journal of Veterinary Research, vol. 69, no. 3, pp. 215-222, 2005.

[39] C. Li, Z. Liu, W. Li et al., "The FSH-HIF- $1 \alpha$-VEGF pathway is critical for ovulation and oocyte health but not necessary for follicular growth in mice," Endocrinology, vol. 161, no. 4, p. 038, 2020.

[40] D. Zhao, Q. Qu, H. Dai et al., "Effects of hypoxia-inducible factor- $1 \alpha$ on endometrial receptivity of women with polycystic ovary syndrome," Molecular Medicine Reports, vol. 17, no. 1, pp. 414-421, 2018.

[41] W. J. Wilson and L. Poellinger, "The dietary flavonoid quercetin modulates HIF- $1 \alpha$ activity in endothelial cells," 
Biochemical and Biophysical Research Communications, vol. 293, no. 1, pp. 446-450, 2002.

[42] F. Pourteymour Fard Tabrizi, F. Hajizadeh-Sharafabad, M. Vaezi, H. Jafari-Vayghan, M. Alizadeh, and V. Maleki, "Quercetin and polycystic ovary syndrome, current evidence and future directions: a systematic review," Journal of Ovarian Research, vol. 13, no. 1, p. 11, 2020.

[43] M. Khorshidi, A. Moini, E. Alipoor et al., "The effects of quercetin supplementation on metabolic and hormonal parameters as well as plasma concentration and gene expression of resist in in overweight or obese women with polycystic ovary syndrome," Phytotherapy Research, vol. 32, no. 11, pp. 2282-2289, 2018.

[44] P. Xu, B.-Y. Huang, J.-H. Zhan et al., "Insulin reduces reaction of follicular granulosa cells to FSH stimulation in women with obesity-related infertility during IVF," Journal of Clinical Endocrinology \& Metabolism, vol. 104, no. 7, pp. 2547-2560, 2019.

[45] M. K. Ediriweera, K. H. Tennekoon, and S. R. Samarakoon, "Role of the PI3K/AKT/mTOR signaling pathway in ovarian cancer: biological and therapeutic significance," Seminars in Cancer Biology, vol. 59, pp. 147-160, 2019.

[46] A. Neisy, F. Zal, A. Seghatoleslam, and S. Alaee, "Amelioration by quercetin of insulin resistance and uterine GLUT4 and ER $\alpha$ gene expression in rats with polycystic ovary syndrome (PCOS)," Reproduction, Fertility and Development, vol. 31, no. 2, pp. 315-323, 2019.

[47] Z. Wang, D. Zhai, D. Zhang et al., "Quercetin decreases insulin resistance in a polycystic ovary syndrome rat model by improving inflammatory microenvironment," Reproductive Sciences, vol. 24, no. 5, pp. 682-690, 2017.

[48] M. F. Heber, S. R. Ferreira, G. A. Abruzzese et al., "Metformin improves ovarian insulin signaling alterations caused by fetal programming," Journal of Endocrinology, vol. 240, no. 3, pp. 431-443, 2019.

[49] K. N. Shah and S. S. Patel, "Phosphatidylinositide 3-kinase inhibition: a new potential target for the treatment of polycystic ovarian syndrome," Pharmaceutical Biology, vol. 54, no. 6, pp. 975-983, 2016.

[50] Y. Huang and X. Zhang, "Luteolin alleviates polycystic ovary syndrome in rats by resolving insulin resistance and oxidative stress," American Journal of Physiology, Endocrinology and Metabolism, vol. 320, no. 6, pp. E1085-E1092, 2021.

[51] X. Dong, J. Fu, X. Yin et al., "Emodin: a review of its pharmacology, toxicity and pharmacokinetics," Phytotherapy Research, vol. 30, no. 8, pp. 1207-1218, 2016.

[52] P. Xu, B.-Y. Huang, J.-H. Zhan et al., "Insulin reduces reaction of follicular granulosa cells to FSH stimulation in women with obesity-related infertility during IVF," Journal of Clinical Endocrinology \& Metabolism, vol. 104, no. 7, pp. 2547-2560, 2019.

[53] C. Kunitomi, M Harada, A Kusamoto et al., "Induction of aryl hydrocarbon receptor in granulosa cells by endoplasmic reticulum stress contributes to pathology of polycystic ovary syndrome," Molecular Human Reproduction, vol. 27, no. 3, Article ID gaab003, 2021.

[54] S. Blauschmidt, T. Greither, K. Lampe, S. Köller, P. Kaltwaßer, and H. M. Behre, "Dipeptidyl peptidase 4 serum activity and concentration are increased in women with polycystic ovary syndrome," Clinical Endocrinology, vol. 87, no. 6, pp. 741-747, 2017.

[55] M. Jensterle, K. Goricar, and A. Janez, "Add on DPP-4 inhibitor alogliptin alone or in combination with pioglitazone improved $\beta$-cell function and insulin sensitivity in metformin treated PCOS," Endocrine Research, vol. 42, no. 4, pp. 261-268, 2017.

[56] A. Kuchina, L. M. Brettner, L. Paleologu et al., "Microbial single-cell RNA sequencing by split-pool barcoding," Science, vol. 371, no. 6531, p. 371, Article ID eaba5257, 2021.

[57] R. Azziz, E. Carmina, Z. Chen et al., "Polycystic ovary syndrome," Nature Reviews Disease Primers, vol. 2, no. 1, Article ID 16057, 2016.

[58] E. R. Vázquez-Martínez, Y. I. Gómez-Viais, E. García-Gómez et al., "DNA methylation in the pathogenesis of polycystic ovary syndrome," Reproduction, vol. 158, no. 1, pp. R27-R40, 2019.

[59] P. Sagvekar, P. Kumar, V. Mangoli, S. Desai, and S. Mukherjee, "DNA methylome profiling of granulosa cells reveals altered methylation in genes regulating vital ovarian functions in polycystic ovary syndrome," Clinical Epigenetics, vol. 11 , no. 1 , p. $61,2019$. 